\title{
Modulating the Diameter of Bulk Single-Walled Carbon Nanotubes Grown by $\mathrm{FeCo} / \mathrm{MgO}$ Catalyst
}

\author{
Zeyao Zhang ${ }^{1,2,4,5}$, Yixi Yao ${ }^{2,3}$, Yan Li ${ }^{2,3,4,{ }^{*}}$ \\ ${ }^{1}$ Peking University Shenzhen Institute, Shenzhen 518057, Guangdong Province, China. \\ ${ }^{2}$ Beijing National Laboratory for Molecular Science, Key Laboratory for the Physics and Chemistry of Nanodevices, State Key \\ Laboratory of Rare Earth Materials Chemistry and Applications, College of Chemistry and Molecular Engineering, \\ Peking University, Beijing 100871, China. \\ ${ }^{3}$ Academy for Advanced Interdisciplinary Studies, Peking University, Beijing 100871, China. \\ ${ }^{4}$ PKU-HKUST ShenZhen-HongKong Institution, Shenzhen 518057, Guangdong Province, China. \\ ${ }^{5}$ College of Engineering, Peking University, Beijing 100871, China.
}

\begin{abstract}
The diameter-controlled growth of single-walled carbon nanotubes (SWNTs) is one of the key issues of SWNT synthesis and application. To guarantee that SWNTs grow with desired diameters, it is necessary to control catalyst size and modulate growth conditions. SWNTs with diameters of 0.9-1.2 nm are highly desirable for near-
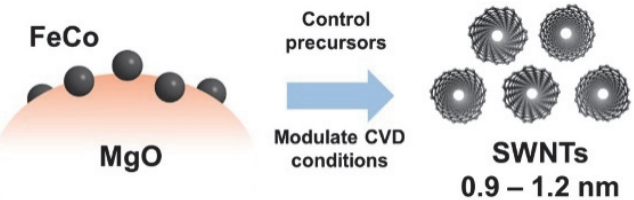

infrared fluorescence bioimaging and serving as effective single-photon sources for the development of quantum devices. Herein, we used an FeCo/MgO catalyst to grow bulk SWNTs with diameters in the range and studied the influence of catalysts and chemical vapor deposition (CVD) growth conditions on the diameter of SWNTs. The preparation of catalyst precursors is a key step in obtaining catalyst nanoparticles of small size. In the impregnation process, we used three different types of metal salts, namely, sulfates, acetates, and nitrates, to prepare the catalysts. The metal sulfates, which exhibit the weakest hydrolysis ability, were found to grow SWNTs with the smallest diameters. Lowering the immersion $\mathrm{pH}$, which suppresses the hydrolysis of metal ions, was also favorable for growing smaller SWNTs. Moreover, the addition of complexing agent molecules such as ethylenediaminetetraacetic acid during the impregnation process, which inhibits the hydrolysis of metal ions as well, further confined the diameter distribution of the resultant SWNTs. During the solution drying process, metal salts hydrolyze into metal hydroxides and oxides. Under mild hydrolysis conditions, the produced hydroxide and oxide particles are smaller and more likely to be uniformly distributed on the surface of the supports. Therefore, it is more favorable to produce catalysts with controlled sizes under mild hydrolysis conditions, which are preferred for diameter control of the resultant SWNTs. In the CVD growth process, we used either ethanol or methane as the carbon source and found that, under our experimental conditions, the SWNTs grown from ethanol had smaller diameters than those from methane. The hydrogen content in the CVD process also affects diameter distribution of SWNTs. As the carbon-tohydrogen ratio decreased, SWNTs with larger diameters disappeared, and the number of SWNTs with smaller diameters increased. During the CVD process, the carbon-to-hydrogen ratio determines the carbon feeding rate to the catalysts. At a low carbon feeding rate, catalysts of large sizes are underfed and unable to grow SWNTs, whereas smaller catalysts are in a favorable condition for growth. Therefore, the average diameter of the SWNTs decreased as the carbon-to-hydrogen ratio decreased.
\end{abstract}

Key Words: Single-walled carbon nanotube; Chemical vapor deposition; Controlled growth; Diameter; Metal catalyst; Precursor; Nano particle

Received: January 28, 2021; Revised: March 2, 2021; Accepted: March 2, 2021; Published online: March 4, 2021.

${ }^{*}$ Corresponding author. Email: yanli@pku.edu.cn; Tel.: +86-10-62756773.

The project was supported by the Shenzhen Basic Research Project (JCYJ20170817113121505), the Shenzhen KQTD Project (KQTD20180411143400981), the National Science and Technology Major Project of the Ministry of Science and Technology of China (2016YFA0201904), the National Natural Science Foundation of China (21631002), and the Beijing National Laboratory for Molecular Sciences (BNLMS-CXTD-202001).

深圳市科技计划项目(JCYJ20170817113121505), 深圳市海外高层次人才创新创业专项资金(KQTD20180411143400981), 科技部国家科技重大专项 (2016YFA0201904), 国家自然科学基金(21631002)及北京分子科学国家研究中心(BNLMS-CXTD-202001)资助

(C) Editorial office of Acta Physico-Chimica Sinica 


\title{
$\mathrm{FeCo} / \mathrm{MgO}$ 催化生长体相单壁碳纳米管的直径调控
}

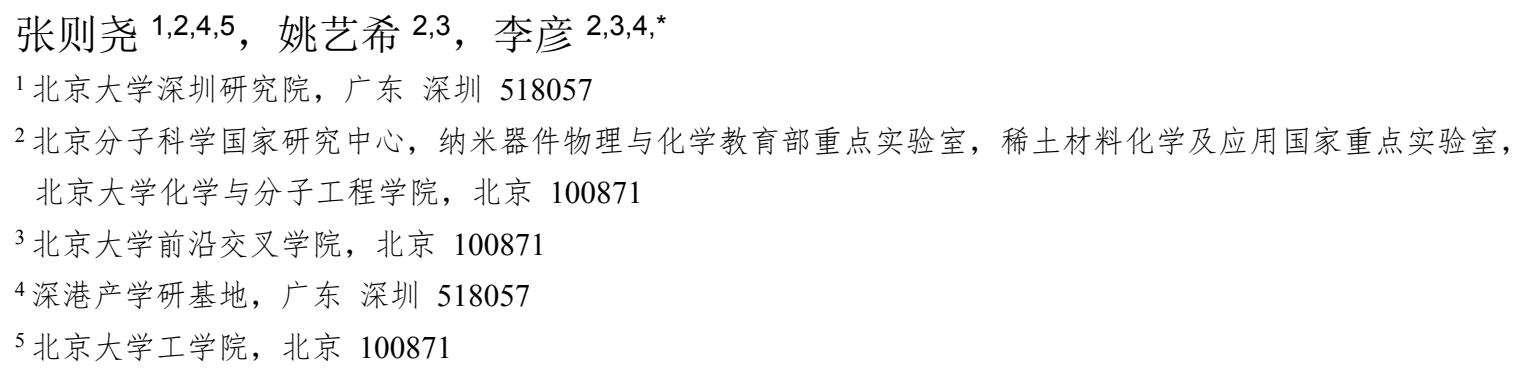

\begin{abstract}
摘要: 单壁碳纳米管的直径可控生长是碳纳米管生长与应用领域的重要问题。直径在0.9-1.2 nm范围内的碳纳米管非常 适合应用于近红外荧光生物成像领域和量子器件单光子光源之中。本文使用 $\mathrm{FeCo} / \mathrm{MgO}$ 催化剂生长出了直径在这一范围 内的体相单壁碳纳米管, 并研究了催化剂制备和CVD生长条件对碳纳米管直径的影响。催化剂前驱体的制备是获得小尺 寸催化剂颗粒的关键步骤。在浸渍过程中, 使用难水解的金属硫酸盐作为前驱体、降低浸渍pH以及加入络合剂分子都会 抑制溶液干燥过程中金属盐的水解, 从而控制催化剂的尺寸, 使其适合于生长出直径可控的单壁碳纳米管。在CVD生长 过程中，使用乙醇作为碳源、使用较低的碳氢比例也有利于小直径碳纳米管的生长。
\end{abstract}

关键词：单壁碳纳米管；化学气相沉积；可控生长；直径；金属催化剂；前驱体；纳米颗粒 中图分类号: 0643

\section{1 引言}

单壁碳纳米管的结构可控生长是其在生物成 像、量子信息处理、微电子学、能量存储与转换等 高端应用中所面临的关键挑战 ${ }^{1-6}$ 。半导体性单壁 碳纳米管的带隙随着管径的增大而减小。因此, 通 过对单壁碳纳米管直径的控制, 就可以调控其带 隙, 从而调节碳纳米管的发光波长和导带、价带的 位置, 以匹配应用的要求。例如, 对于生物深层组 织的苂光成像而言, 苂光探针分子的理想发射波 长应处于第二近红外窗口 $(1000-1400 \mathrm{~nm})^{7}$ 。与这 一发射波长范围相对应的是直径在0.8-1.2 $\mathrm{nm}$ 之 间的单壁碳纳米管。再比如, 少量共价功能化基团 修饰的单壁碳纳米管具有很高的室温单光子发射 纯度, 是极具应用前景的单光子光源 ${ }^{4}$ 。在量子信 息处理器件中, 单光子光源的理想发射波长是 $1550 \mathrm{~nm}$ 。由于共价修饰带来的缺陷一般会使单壁 碳纳米管的发射峰红移 100-300 meV, 因此原始发 射波长在1100-1400 $\mathrm{nm}$ 的单壁碳纳米管最适合于 这一应用。与这一发射波长范围相对应的是直径 在0.9-1.2 nm之间的单壁碳纳米管。

在化学气相沉积法 (Chemical vapor deposition, CVD)生长碳纳米管的过程中, 控制单 壁碳纳米管的直径主要有两个关键, 其一是对催 化剂尺寸分布的调控, 其二是对 CVD生长条件的 控制 ${ }^{8-11}$ 。首先, 催化剂的尺寸分布会直接影响碳 纳米管的直径分布。 $\mathrm{Li}$ 等人仔细研究了不同生长
条件下, 催化剂颗粒的尺寸分布和单壁碳纳米管 直径分布的变化情况 ${ }^{12}$ 。他们发现, 碳纳米管的直 径分布总是跟随着催化剂颗粒的尺寸分布而变 化。因此, 合成尺寸均一可控的催化剂是碳纳米管 直径可控生长的关键。催化剂载体通常可以发挥 稳定催化剂尺寸、限制其团聚的作用。例如, $\mathrm{He}$ 等人发现, 利用 $\mathrm{MgO}$ 载体与 $\mathrm{Co}$ 催化剂之间的晶格 错配, 可以有效地 $\mathrm{Co}$ 颗粒的尺寸, 从而生长出直 径较小且分布较窄的单壁碳纳米管 ${ }^{13,14}$ 。沸石 ${ }^{15,16}$ 、 介孔氧化硅 ${ }^{17,18}$ 等多孔载体, 也可以通过纳米尺寸 的孔道限制催化剂的大小。

在CVD反应过程中, 碳源的种类 19,20 、分压 ${ }^{21,22}$ 、 碳源与氢气的比例 23,24 等条件均会影响碳纳米管 的直径分布。不同种类的碳源分子在催化剂上裂 解形成碳物种的能力各不相同。碳供给能力的差 异, 会影响催化剂生长碳纳米管的直径。碳源的分 压也会直接影响催化剂上碳供给的速率。Liu等人 发现, 随着乙烷碳源分压降低, 碳纳米管的平均直 径逐渐减小 21 。他们认为, 在一定的供碳速率下, 催化剂颗粒存在着某一最优尺寸。尺寸过大或过 小的催化剂均不能有效生长碳纳米管。由于小尺 寸的催化剂催化活性更高, 在碳源分压降低时, 适 合于生长碳纳米管的催化剂尺寸也随之减小, 因 此会生长出直径更小的碳纳米管。碳源分压还可 以通过影响催化剂上碳纳米管的成核时间来影响 其直径。Chen等人发现, 随着一氧化碳碳源压力 
的升高, 生长出的碳纳米管直径逐渐减小 22 。他们 认为, 碳源压力较高时, 催化剂颗粒只需要较短的 时间就可以达到溶碳饱和, 开始碳纳米管的成核、 生长过程。由于CVD过程中催化剂颗粒往往会因 奥斯特瓦尔德熟化而不断长大, 因此更短的成核 时间就意味着成核时催化剂的尺寸更小, 因此最 终碳纳米管的直径也更小。在使用烃类、醇类碳源 生长碳纳米管时, 往往会加入氢气, 调节生长情 况。由于氢气是这些碳源裂解反应的产物之一, 因 此氢气的浓度会影响碳源裂解反应的平衡。同时, 氢气对碳纳米管有一定的刻蚀作用, 也会影响其 直径分布 ${ }^{23,24}$ 。

$\mathrm{Fe} 、 \mathrm{Co}$ 均是生长碳纳米管常用的催化 剂。Robertson等人发现, $\mathrm{Fe}$ 与 $\mathrm{Co}$ 在催化生长碳纳 米管时, 存在一定的协同作用, 可以提高碳纳米管 的生长效率 ${ }^{25}$ 。Maruyama等人使用多孔的USY型 沸石作为载体, 负载 $\mathrm{FeCo}$ 双金属催化剂, 生长出 了直径在 $0.8-1.2 \mathrm{~nm}$ 的小直径单壁碳纳米管 ${ }^{15}$ 。沸 石的多孔结构可能起到了限制催化剂尺寸的作 用。但沸石载体较难除去, 这给碳纳米管的应用带 来了一定的不便。

本文中, 我们使用 $\mathrm{MgO}$ 作为载体负载 $\mathrm{FeCo}$ 催 化剂生长单壁碳纳米管, 并对其直径进行调控。 $\mathrm{FeCo}$ 催化剂可以高效地生长单壁碳纳米管。 $\mathrm{MgO}$ 载体使用稀酸浸泡即可去除, 非常简便, 便于后续 应用中碳纳米管的溶液分散。我们研究了前驱体 浸渍过程中 Fe 、 Co 金属盐的种类、浸渍 $\mathrm{pH}$ 、络合 试剂等条件和 $\mathrm{CVD}$ 生长过程中碳源种类、碳氢比 例、生长温度等条件对于生长出的碳纳米管直径 的影响。在优化的条件下, 使用 $\mathrm{FeCo} / \mathrm{MgO}$ 催化剂 生长出直径在 $0.9-1.2 \mathrm{~nm}$ 的单壁碳纳米管。

\section{2 实验部分}

\section{1 试剂与仪器}

实验使用的 $\mathrm{Fe}\left(\mathrm{NO}_{3}\right)_{3} \cdot 9 \mathrm{H}_{2} \mathrm{O}$ (纯度 $98.5 \%$ )、 $\mathrm{Co}\left(\mathrm{SO}_{4}\right)_{2} \cdot 7 \mathrm{H}_{2} \mathrm{O}$ (纯度 $99.5 \%$ )、 $\mathrm{Co}\left(\mathrm{NO}_{3}\right)_{2} \cdot 6 \mathrm{H}_{2} \mathrm{O}$ (纯度 $98.5 \%$ )、 $\mathrm{Co}\left(\mathrm{CH}_{3} \mathrm{COO}\right)_{2} \cdot 4 \mathrm{H}_{2} \mathrm{O}$ (纯度 $99.5 \%$ ) 购自西 陇化工公司; $\left(\mathrm{NH}_{4}\right)_{2} \mathrm{Fe}\left(\mathrm{SO}_{4}\right)_{2} \cdot 6 \mathrm{H}_{2} \mathrm{O}$ (纯度 $99.5 \%$ )、 轻质氧化镁(纯度 $98.5 \%$ ) 购自国药试剂公司; $\mathrm{Fe}\left(\mathrm{CH}_{3} \mathrm{COO}\right)_{2} \cdot 4 \mathrm{H}_{2} \mathrm{O}$ (纯度 $95 \%$ ) 购自麦克林生化试 剂公司; 乙二胺四乙酸(分析纯)、无水乙醇(分析 纯)购自北京北化精细化学公司; 脱氧胆酸钠 (BioXtra级, 纯度 $98 \%$ ) 购自Sigma公司; 氩气、氢 气、甲烷(纯度 $99.999 \%$ )购自北京华元气体公司; 超 纯水(18.2 M $\Omega$ )由 Millipore Simplicity超纯水机 制备。
碳纳米管的生长在自行搭建的气相沉积系统 中进行。使用的管式炉(天津中环实验电炉有限公 司)恒温区长度为 $6 \mathrm{~cm}$; 石英管长度为 $1.0 \mathrm{~m}$, 内径 为 $2.1 \mathrm{~cm}$ 。实验使用的拉曼光谱仪为Horiba JobinYvon公司的LabRAM ARAMIS型拉曼光谱仪, 配 有 $532 、 633 \mathrm{~nm}$ 两个波长的激光。测试时激光光斑 的直径约为 $2 \mu \mathrm{m}$ 。吸收光谱仪为Perkin Elmer公司 的 Lambda 750型吸收光谱仪。X射线粉末衍射 (XRD) 测试是在PANalytical公司的X-Pert3 型粉末 $\mathrm{X}$ 射线衍射仪上进行的。测试使用 $\mathrm{Cu} K_{a}$ 辐射 $(\lambda=$ $0.15406 \mathrm{~nm}, 40 \mathrm{kV}, 40 \mathrm{~mA}$ )。

\section{2 实验方法}

\subsection{1 制备催化剂前驱体}

分别称取 $0.83 \mathrm{mmol} \mathrm{Fe}$ 盐和 $\mathrm{Co}$ 盐, 并分别溶解 于 $7.0 \mathrm{~mL}$ 超纯水中。称取 $3.33 \mathrm{~g}(83 \mathrm{mmol})$ 轻质 $\mathrm{MgO}$ 于 $33 \mathrm{~mL}$ 超纯水中, 超声 $5 \mathrm{~min}$ 至均匀分散。在搅拌 下, 先后将 $\mathrm{Fe}$ 盐、 $\mathrm{Co}$ 盐溶液滴加至 $\mathrm{MgO}$ 悬浊液中 (此时悬浊液中 $\mathrm{Co} 、 \mathrm{Fe}$ 浓度均为 $0.16 \mathrm{~mol} \cdot \mathrm{L}^{-1}$ )。将悬 浊液搅拌 $10 \mathrm{~min}$, 再煮沸 $30 \mathrm{~min}$, 冷却后抽滤, 并 用超纯水、无水乙醇各洗涤两次, 在烘箱中 $120^{\circ} \mathrm{C}$ 干燥 $12 \mathrm{~h}$, 即可得 $\mathrm{Mg}: \mathrm{Fe}: \mathrm{Co}$ 摩尔比为 $100: 1: 1$ 的 $\mathrm{FeCo} / \mathrm{MgO}$ 催化剂前驱体。

\subsubsection{CVD生长单壁碳纳米管}

1)称取约 $50 \mathrm{mg}$ 催化剂前驱体于 $7 \mathrm{~cm} \times 0.5 \mathrm{~cm}$ 的瓷舟中, 推入管式炉中央。2)灼烧: 在空气气氛 中升温至 $700{ }^{\circ} \mathrm{C}$, 灼烧 $3 \mathrm{~min}$; 通入 $\mathrm{Ar} 200 \mathrm{sccm}$ (standard cubic centimeter per minute) $4 \mathrm{~min}$, 以排 出空气, 同时将炉温升至 $900{ }^{\circ} \mathrm{C}$ 。3)还原: 升温至 $900{ }^{\circ} \mathrm{C}$ 后, 通入Ar $100 \mathrm{sccm} 、 \mathrm{H}_{2} 50 \mathrm{sccm}$, 保持 3 $\mathrm{min}$, 以还原得到金属催化剂。4)生长: 在 $200 \mathrm{sccm}$ 的 $\mathrm{Ar}$ 保护下将炉温调整至实验设计的生长温度, 使 $200 \mathrm{sccm}$ 的Ar经过乙醇鼓泡器 (冰水浴恒温) 后通 入石英管内, 同时通入 $\mathrm{H}_{2} 30 \mathrm{sccm}$, 或通入 $\mathrm{CH}_{4} 325$ $\mathrm{sccm} 、 \mathrm{H}_{2} 30 \mathrm{sccm}$, 开始生长; 生长过程持续 $15 \mathrm{~min}$ 。 5)降温: 生长结束后, 依次在 $\mathrm{H}_{2} 、 \mathrm{Ar}$ 氛围的保护下 降至室温。

\section{3 结果与讨论}

首先, 我们比较了分别使用两种常用的碳 源一甲烷和乙醇, 生长出的碳纳米管在直径上的 差别。图1中分别展示了使用铁、钴的硝酸盐、乙 酸盐和硫酸盐催化剂前驱体生长出的碳纳米管拉 曼光谱。比较上述样品拉曼光谱图中的径向呼吸 模(Radial breathing mode, RBM)峰位可以发现, 使 用乙醇生长的碳纳米管在较低波数的 RBM峰总是 弱于使用甲烷生长的碳纳米管样品, 而在较高波 
数的RBM峰相对较强。这意味着乙醇生长的碳纳 米管直径小于甲烷生长的样品。这样的现象可能 是由于乙醇与甲烷在催化生长过程中供碳速率的 差异所造成的。碳纳米管的催化生长一般遵循气 液固(Vapor-liquid-solid)机理, 即气相的碳源分子 在液相的催化剂上裂解, 碳原子溶解进入催化剂 中, 直至达到饱和、开始析出, 形成碳纳米管的帽 端, 进而生长碳纳米管。相比甲烷分子而言, 乙醇 分子相对更容易裂解, 供碳速率较高。因此在乙醇 充当碳源时，催化剂可以在更短的时间内达到溶 碳量饱和, 开始碳纳米管的成核生长过程。这就意 味着催化剂颗粒在开始生长之前, 发生团聚、长大 的时间也更短, 因此可以减小碳纳米管的平均直 径。提高供碳速率时碳纳米管平均直径减小的现 象, 在使用 $\mathrm{Co}{ }^{18} 、 \mathrm{Ni}^{26}$ 催化剂生长碳纳米管的研 究中也有报道。因为在多种不同的催化剂制备条 件以及碳纳米管生长条件下, 乙醇碳源相比甲烷 碳源总是更倾向于生长小直径的碳纳米管, 所以 在后续实验中, 我们均采用乙醇作为碳源生长碳 纳米管。

a)
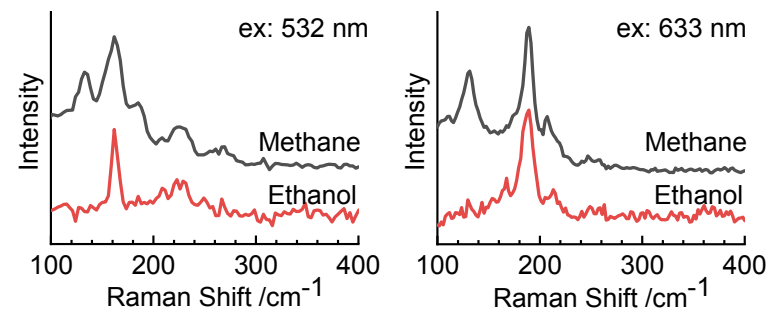

b)
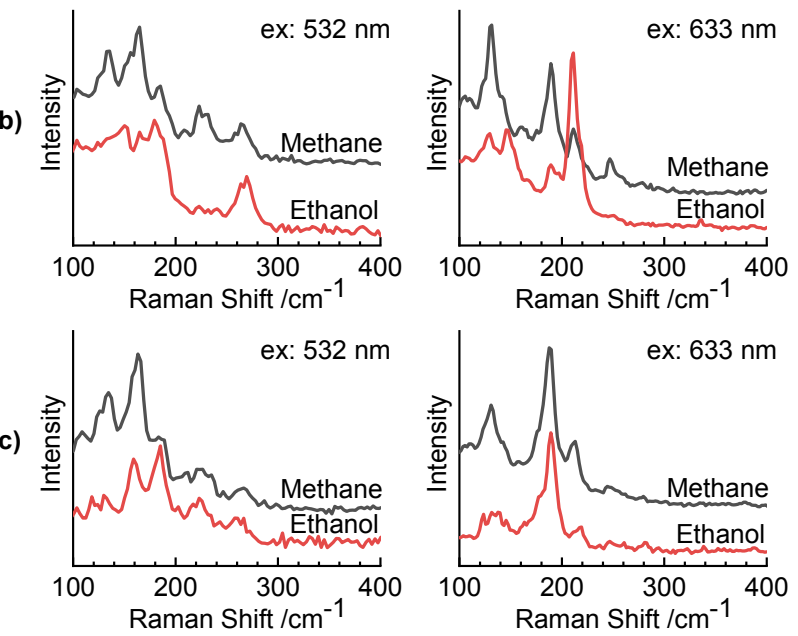

图 1 碳源对碳纳米管直径的影响

Fig. 1 The effect of carbon source on the diameter of single-walled carbon nanotubes (SWNTs).

The SWNTs were grown using various catalyst precursors, including nitrates (a), acetates (b), and sulfates (c) of Fe and Co. The spectra of SWNTs grown using either ethanol or methane as the carbon source were compared in each panel.
选定碳源后, 我们研究了催化剂前驱体的种 类对于碳纳米管直径的影响。我们分别使用硝酸 铁与硝酸钴、硫酸亚铁铵与硫酸钴、硫酸亚铁铵与 乙酸钴, 制备 $\mathrm{Mg}: \mathrm{Fe}: \mathrm{Co}$ 摩尔比为 $100: 1: 1$ 的 $\mathrm{FeCo} / \mathrm{MgO}$ 催化剂前驱体, 并在相同条件下生长碳 纳米管。三种前驱体生长的碳纳米管分别以硝酸 盐、硫酸盐、乙酸盐标记。拉曼光谱中, 使用硫酸 盐前驱体生长的碳纳米管RBM峰大致出现在更高 波数(图2)。为了更仔细地分析上述样品中碳纳米 管的直径分布情况, 我们对上述拉曼光谱进行分 峰拟合(图2)。我们选取 RBM峰集中的100-300 $\mathrm{cm}^{-1}$ 区域进行分峰拟合, 峰形采用洛伦兹峰。从每 个分出的RBM峰的峰位, 可以计算出其对应的单 壁碳纳米管直径。考虑到我们的样品是负载型催 化剂生长的体相单壁碳纳米管, 这里选用的RBM 峰位 $\omega\left(\mathrm{cm}^{-1}\right)$ 与单壁碳纳米管管径 $d_{\mathrm{t}}(\mathrm{nm})$ 之间的 ex: $532 \mathrm{~nm}$

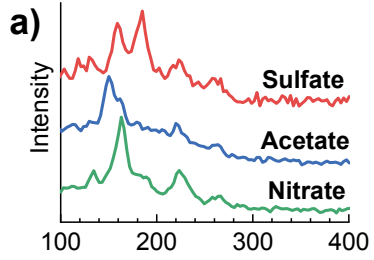

c)

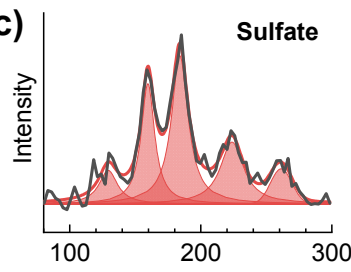

e)
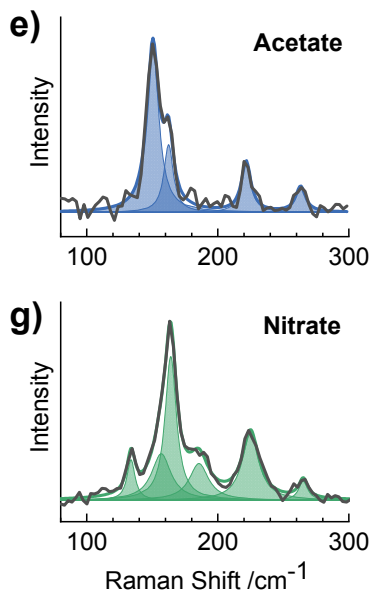

ex: $633 \mathrm{~nm}$
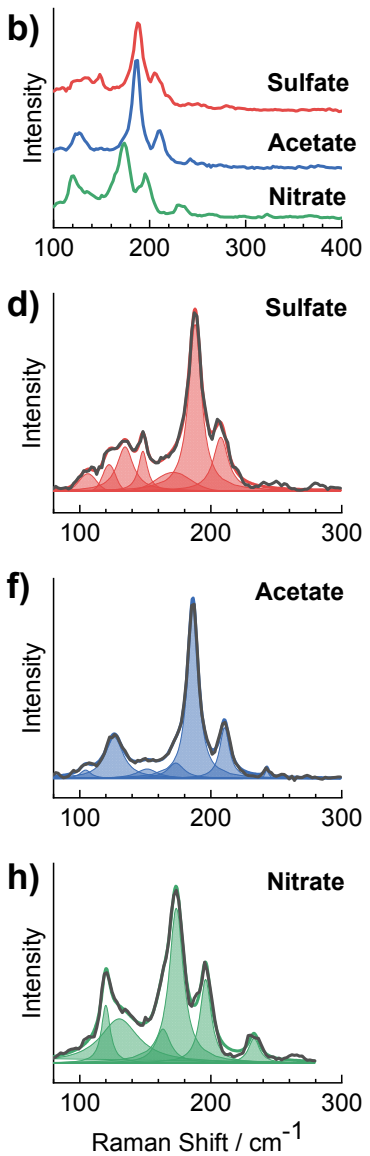

图 2 前驱体盐种类对碳纳米管直径的影响

Fig. 2 The influence of precursor salt type on the diameter of SWNTs.

Raman spectra $(a, b)$ of SWNTs grown using the acetates, sulfates, and nitrates as the catalyst precursors and the multi-peak fitting analysis results $(c-h)$ of each spectrum are shown respectively. 
关系式为 $\omega=227.0 / d_{\mathrm{t}}-0.3^{27,28}$ 。 RBM峰的峰面积 与其对应单壁碳纳米管的含量直接相关。由于样 品中单壁碳纳米管的直径主要集中在 1-2 $\mathrm{nm}$ 之 间, 因此我们在分析时假设各直径的单壁碳纳米 管RBM声子的拉曼散射截面均相同, 并根据各 RBM峰的峰面积计算了相应直径的单壁碳纳米管 的相对含量、加权平均直径和加权平均标准差(图 3)。从这些数据中, 我们可以更清楚地发现, 在三 种前驱体生长的样品中, 硫酸盐生长的单壁碳纳 米管直径最小 $(1.25 \pm 0.23 \mathrm{~nm}, 532 \mathrm{~nm}$ 激发; $1.36 \pm$ $0.28 \mathrm{~nm}, 633 \mathrm{~nm}$ 激发)。

这一现象可能是由不同前驱体盐的水解难易 程度差异所造成的。在溶液蒸发过程, 金属盐通常 会发生水解, 形成氢氧化物或氧化物 ${ }^{29}$ 。前驱体水 解程度越剧烈、团聚情况越严重时, 最终形成的催 化剂颗粒尺寸也就越大, 因此会造成碳纳米管直 径的增大。从水解的难易程度来说, 由于硫酸不易 挥发, 而硝酸和乙酸都有较高的挥发性, 因此硫酸 盐在溶液蒸发过程中最难水解, 而乙酸盐与硝酸 盐都会发生更为严重的水解, 颗粒尺寸更大。我们 对上述所有实验中浸渍制备的催化剂前驱体和还 原后得到的催化剂进行了XRD测试。得到的谱图 中仅有 $\mathrm{MgO}$ 与 $\mathrm{Mg}(\mathrm{OH})_{2}$ 的衍射峰, 没有任何 $\mathrm{Fe} 、 \mathrm{Co}$ 物相的衍射峰。这说明 $\mathrm{Fe} 、 \mathrm{Co}$ 均匀分散在了 $\mathrm{MgO}$ 载体表面, 没有发生明显团聚。由于 Fe、Co物相

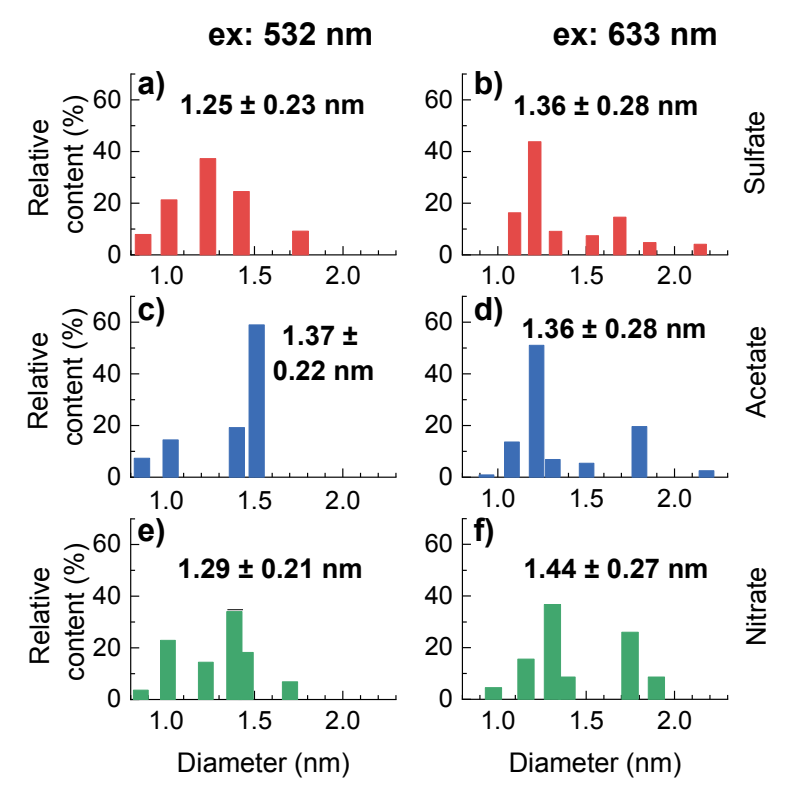

图 3 三种不同前驱体盐生长出碳纳米管的直径分布

Fig. 3 Diameter distribution of SWNTs grown from sulfate (a, b), acetate (c, d), and nitrate (e, f) precursors.

The relative abundances at each tube diameter were calculated based on the RBM peak areas from multi-peak fitting analysis of the Raman spectra.
的晶粒尺寸较小, 并且可能晶化程度较低, 所以在 XRD谱图中不能观测到 $\mathrm{Fe} 、 \mathrm{Co}$ 物相的衍射峰。基 于上述结果, 在后续实验中, 我们均采用硫酸盐前 驱体生长碳纳米管。

浸渍过程的 $\mathrm{pH}$ 同样会影响碳纳米管的直径分 布。在使用硫酸盐前驱体浸渍时, 当 $\mathrm{pH}$ 由10-11降 低至9-10时, 生长出的碳纳米管RBM峰位明显更 集中于高波数区域(图4), 管径变减小。这很可能 是由于 $\mathrm{pH}$ 减小抑制了金属盐的水解, 造成催化剂 尺寸减小所致。但是, 由于 $\mathrm{MgO}$ 载体在 $\mathrm{pH}$ 低于 9 时 即开始溶解, 限制了 $\mathrm{pH}$ 的调节范围, 因此并未能 在更低 $\mathrm{pH}$ 下进行浸渍实验。

我们进而研究了CVD生长过程中, 乙醇与氢 气的比例对碳纳米管管径的影响。我们将 $\mathrm{Ar}$ 载乙 醇的流量固定在 $200 \mathrm{sccm}$, 在0-80 sccm的范围内 调节氢气流量。随着氢气流量由 0 开始逐渐增加, 拉曼光谱中 $100-180 \mathrm{~cm}^{-1}$ 的 RBM峰逐渐减弱或消 失(对应直径在 2.4-1.3 $\mathrm{nm}$ 的单壁碳纳米管), 而 200 $\mathrm{cm}^{-1}$ 以上的 RBM峰逐渐增强(对应直径小于 $1.2 \mathrm{~nm}$ 的单壁碳纳米管, 如图5所示)。这说明, 随着 CVD
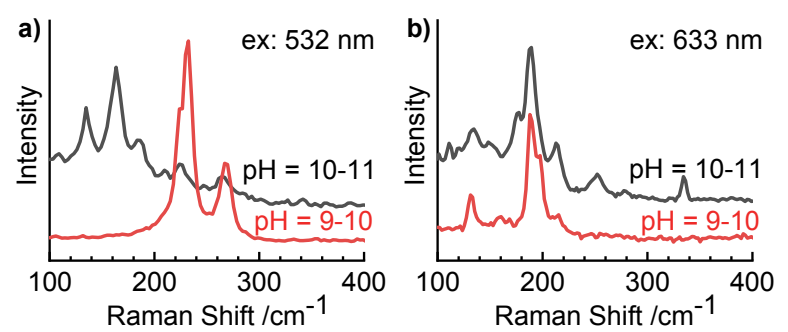

图 4 浸渍 $\mathrm{pH}$ 对碳纳米管直径的影响

Fig. 4 The influence of impregnating $\mathrm{pH}$ on the diameter of SWNTs.

Raman spectra of SWNTs grown by impregnating the sulfate catalyst precursors at different $\mathrm{pH}$.

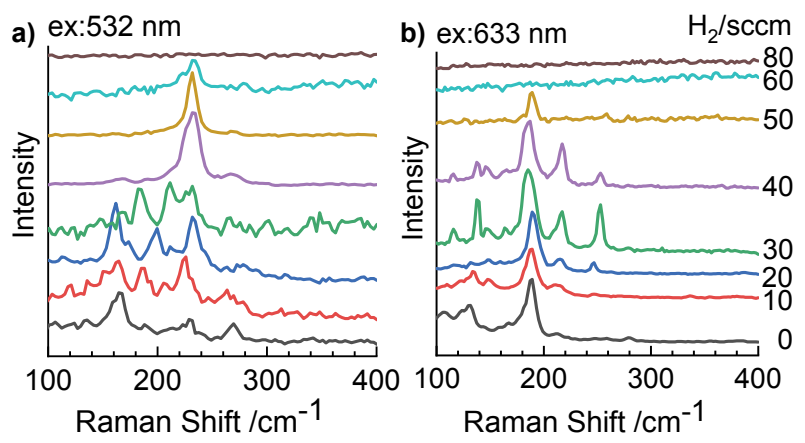

图 5 碳氢比例对碳纳米管直径的影响

Fig. 5 The influence of carbon-hydrogen ratio on the diameter of SWNTs.

Raman spectra of SWNTs grown under a fixed ethanol flow and various hydrogen flows. 
气氛中碳氢比的降低, 生长的碳纳米管直径逐渐 减小。这可能是由于氢气的相对含量影响了供碳 速率, 从而影响了生长碳纳米管的直径。由于催化 剂的尺寸越小, 其催化活性就越强, 因此在一定的 供碳速率下, 只有尺寸合适、催化能力与供碳速率 相匹配的催化剂颗粒, 才能催化生长碳纳米管 ${ }^{21}$ 。 对于尺寸过小的催化剂而言, 由于催化剂活性过 高, 生长出的石墨化碳层很快就会将催化剂包覆, 阻隔催化剂接触新的碳源分子, 因而发生失活; 而 对于尺寸过大的催化剂, 由于催化活性不足, 难以 得到足够的碳, 达到饱和, 因此难以催化碳纳米管 成核生长。随着供碳速率的降低, 适宜生长碳纳米 管的催化剂尺寸也减小, 因此当氢气流量增加、供 碳速率降低时, 生长的碳纳米管直径也逐渐减小。 当氢气流量过高时 (大于 $40 \mathrm{sccm}$ ), 由于氢气的刻 蚀作用, 碳纳米管的生长受到抑制, 拉曼光谱中的 RBM峰明显减弱, 直至消失。上述实验中氢气流 量为 $30 \mathrm{sccm}$ 时, 生长出的碳纳米管直径最小。这 一条件下生长出的碳纳米管主要集中在 0.9-1.2 $\mathrm{nm}$ 的范围之内, 但仍然含有一些更大直径的碳纳 米管 $(1.6 \mathrm{~nm}$ 左右 $)$ 。

最后, 我们发现在制备催化剂前驱体时, 加入 具有配位络合作用的分子乙二胺四乙酸(EDTA), 研究其对于碳纳米管直径分布的影响。我们在催 化剂浸渍步骤前, 向金属盐溶液中加入了 5 倍于金 属摩尔量的EDTA, 并在相同的条件下制备催化 剂、生长碳纳米管。与未加入EDTA的生长结果相 比, 加入EDTA后生长的碳纳米管拉曼光谱中的 RBM峰向高波数区域集中(图6a-f)。经分峰拟合分 析, 我们计算出, 在未加入EDTA时, 样品中单壁 碳纳米管的加权平均直径、加权平均标准差为 $1.08 \pm 0.09 \mathrm{~nm}(532 \mathrm{~nm}$ 激发)、1.26 $\pm 0.28 \mathrm{~nm}$ (633 $\mathrm{nm}$ 激发)。其中, 直径在 $0.9-1.2 \mathrm{~nm}$ 的范围之内的 单壁碳纳米管含量小于 $80 \%$ (532 nm激发为78\%、 $633 \mathrm{~nm}$ 激发为 $79 \%$ )。而加入EDTA后生长的单壁碳 纳米管的加权平均直径、加权平均标准差为 $0.99 \pm$ $0.05 \mathrm{~nm}(532 \mathrm{~nm}$ 激发)、1.10 $\pm 0.16 \mathrm{~nm}(633 \mathrm{~nm}$ 激 发)。其中, 直径在 0.9-1.2 nm的范围之内的单壁碳 纳米管占全部激发出的碳纳米管的含量接近 $100 \%$ $(532 \mathrm{~nm}$ 激发为 $100 \% 、 633 \mathrm{~nm}$ 激发为 $97 \%)$ 。为更充 分地表征这一样品中碳纳米管的管径分布, 我们 将这一样品分散在 $1 \%$ 的脱氧胆酸钠水溶液中, 测 试其紫外可见近红外吸收光谱(图6g)。光谱中 $\mathrm{S} 11$ 区域主要的吸收峰来自 $(10,5) 、(8,7) 、(8,6) 、(7,6)$ 、 $(9,4)$ 手性碳纳米管, 其对应直径为 1.04-0.88 nm。 这部分碳纳米管可以与拉曼光谱中得到的直径范 a)

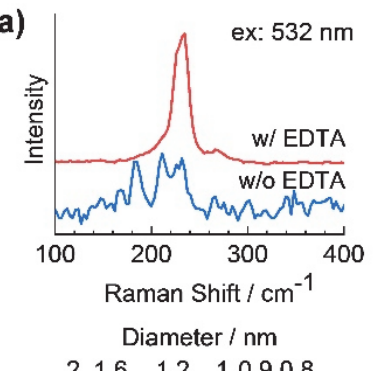

c)

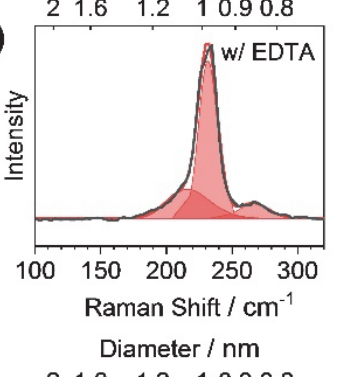

e)

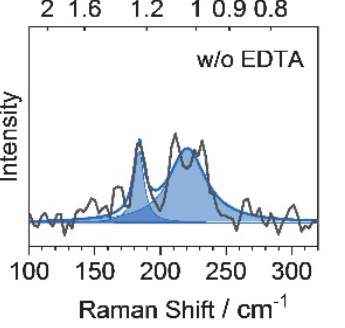

g) b) $\quad$ ex: $633 \mathrm{~nm}$

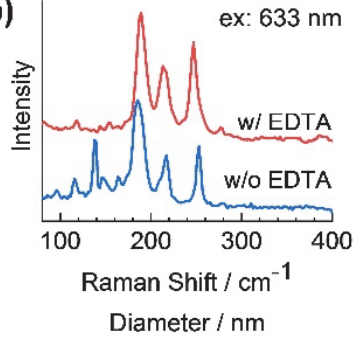

d)

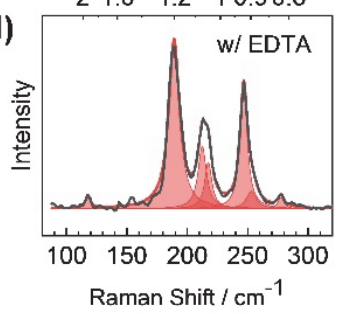

Diameter $/ \mathrm{nm}$

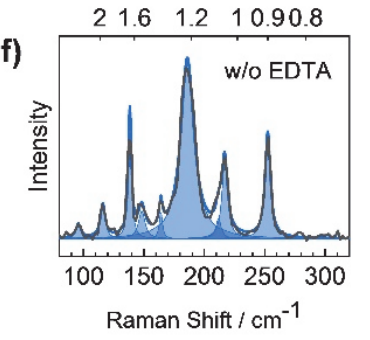

图 6 EDTA 络合剂对碳纳米管直径的影响

Fig. 6 The effect of EDTA complexing agent on the diameter of SWNTs.

Raman spectra $(a, b)$ of SWNTs grown with or without EDTA as the complexing agent during the impregnation and the multi-peak fitting analysis results $(\mathrm{c}-\mathrm{f})$ of each spectrum, respectively. The absorption spectrum of the grown SWNTs is shown in panel $(\mathrm{g})$.

围相吻合。由于 $1400 \mathrm{~nm}$ 以上水分子出现吸收峰, 因此噪音很大, 难以获得有效信号。在我们的吸收 光谱表征中, 没有获得直径在 1.0-1.2 nm范围碳纳 米管的信号。此外, 在 $1000 \mathrm{~nm}$ 附近, 还有较弱的 来自 $(7,5) 、(6,5)$ 手性碳纳米管的吸收峰, 其直径在 $0.8 \mathrm{~nm}$ 左右。EDTA对直径的调控作用可能源于其 与金属离子的配位络合能力。EDTA与 $\mathrm{Fe} 、 \mathrm{Co}$ 等金 属离子配位形成络合物时, 可以抑制其水解沉淀, 因此可能起到了限制催化剂颗粒尺寸的作用。加 入EDTA络合剂生长出的单壁碳纳米管直径几乎 全部落在 0.9-1.2 nm的范围之内。该碳纳米管样品 的直径分布可以很好地满足近红外苂光生物成像 
和量子器件单光子光源对于碳纳米管样品的要 求。

\section{4 结论}

我们使用 $\mathrm{MgO}$ 作为载体负载 $\mathrm{FeCo}$ 催化剂生长 单壁碳纳米管, 并研究了催化剂制备和CVD生长 条件对碳纳米管直径的影响。使用干燥过程中水 解能力较弱的硫酸盐前驱体生长得到的碳纳米管 直径小于使用硝酸盐和乙酸盐前驱体生长的结 果。降低浸渍pH、加入络合剂EDTA也有利于抑制 前驱体的水解、控制催化剂的尺寸, 从而生长出直 径较小的单壁碳纳米管。在CVD生长过程中, 使用 乙醇作为碳源生长出的碳纳米管直径小于使用甲 烷作为碳源生长的结果。较低的碳氢比例也有利 于小直径碳纳米管的生长。在优化的条件下, 直径 在0.9-1.2 nm范围之内的单壁碳纳米管含量很高。 这一直径分布可以很好地满足近红外苂光生物成 像和量子器件单光子光源等应用对于单壁碳纳米 管样品的要求。

\section{References}

(1) De Volder, M. F. L.; Tawfick, S. H.; Baughman, R. H.; Hart, A. J. Science 2013, 339, 535. doi: 10.1126/science. 1222453

(2) Hong, G.; Diao, S.; Antaris, A. L.; Dai, H. Chem. Rev. 2015, 115, 10816. doi: 10.1021/acs.chemrev.5b00008

(3) Zhang, R.; Zhang, Y.; Wei, F. Chem. Soc. Rev. 2017, 46, 3661. doi: 10.1039/C7CS00104E

(4) He, X.; Htoon, H.; Doorn, S. K.; Pernice, W. H. P.; Pyatkov, F.; Krupke, R.; Jeantet, A.; Chassagneux, Y.; Voisin, C. Nat. Mater. 2018, 17, 663. doi: 10.1038/s41563-018-0109-2

(5) Rao, R.; Pint, C. L.; Islam, A. E.; Weatherup, R. S.; Hofmann, S.; Meshot, E. R.; Wu, F.; Zhou, C.; Dee, N.; Amama, P. B.; et al. ACS Nano 2018, 12, 11756. doi: 10.1021/acsnano.8b06511

(6) Zhang, S., Zhang, N., Zhang, J. Acta Phys. -Chim. Sin. 2020, 36, 54. [张树辰, 张娜, 张锦. 物理化学学报, 2020, 36, 54.] doi: 10.3866/PKU.WHXB201907021

(7) Welsher, K.; Sherlock, S. P.; Dai, H. Proc. Natl. Acad. Sci. U. S. A. 2011, 108, 8943. doi: 10.1073/pnas.1014501108

(8) Li, M.; Liu, X.; Zhao, X.; Yang, F.; Wang, X.; Li, Y. Top. Curr. Chem. 2017, 375, 29. doi: 10.1007/s41061-017-0116-9

(9) He, M.; Zhang, S.; Wu, Q.; Xue, H.; Xin, B.; Wang, D.; Zhang, J. $A d v$. Mater. 2019, 31, 1800805. doi: 10.1002/adma.201800805

(10) Yang, F.; Wang, M.; Zhang, D.; Yang, J.; Zheng, M.; Li, Y. Chem. Rev.
2020, 120, 2693. doi: 10.1021/acs.chemrev.9b00835

(11) Zhang, Z.; Yao, Y.; Li, Y. Sci. China Chem. 2020, 50, 1188. [张则尧, 姚艺 希, 李彦. 中国科学: 化学, 2020, 50, 1188.] doi: 10.1360/SSC-2020-0115

(12) Peng, F.; Luo, D.; Sun, H.; Wang, J.; Yang, F.; Li, R.; Yang, J.; Li, Y. Chin. Sci. Bull. 2013, 58, 433. doi: 10.1007/s11434-012-5588-y

(13) He, M.; Jiang, H.; Liu, B.; Fedotov, P. V.; Chernov, A. I.; Obraztsova, E. D.; Cavalca, F.; Wagner, J. B.; Hansen, T. W.; Anoshkin, I. V.; et al. Sci. Rep. 2013, 3, 1460. doi: 10.1038/srep01460

(14) He, M.; Jiang, H.; Kauppi, I.; Fedotov, P. V.; Chernov, A. I.; Obraztsova, E. D.; Cavalca, F.; Wagner, J. B.; Hansen, T. W.; Sainio, J.; et al. J. Mater. Chem. A 2014, 2, 5883. doi: 10.1039/C3TA15325H

(15) Maruyama, S.; Kojima, R.; Miyauchi, Y.; Chiashi, S.; Kohno, M. Chem. Phys. Lett. 2002, 360, 229. doi: 10.1016/S0009-2614(02)00838-2

(16) Miyauchi, Y.; Chiashi, S.; Murakami, Y.; Hayashida, Y.; Maruyama, S. Chem. Phys. Lett. 2004, 387, 198. doi: 10.1016/j.cplett.2004.01.116

(17) Lim, S.; Ciuparu, D.; Pak, C.; Dobek, F.; Chen, Y.; Harding, D.; Pfefferle, L.; Haller, G. J. Phys. Chem. B 2003, 107, 11048. doi: 10.1021/jp0304778

(18) Chen, Y.; Ciuparu, D.; Lim, S.; Yang, Y.; Haller, G. L.; Pfefferle, L. J. Catal. 2004, 226, 351. doi: 10.1016/j.jcat.2004.04.022

(19) Wang, B.; Poa, C. H. P.; Wei, L.; Li, L.-J.; Yang, Y.; Chen, Y. J. Am. Chem. Soc. 2007, 129, 9014. doi: 10.1021/ja070808k

(20) He, M.; Magnin, Y.; Jiang, H.; Amara, H.; Kauppinen, E. I.; Loiseau, A.; Bichara, C. Nanoscale 2018, 10, 6744. doi: 10.1039/c7nr09539b

(21) Lu, C.; Liu, J. J Phys Chem B 2006, 110, 20254. doi: 10.1021/jp0632283

(22) Wang, B.; Wei, L.; Yao, L.; Li, L.-J.; Yang, Y.; Chen, Y. J. Phys. Chem. C 2007, 111, 14612. doi: 10.1021/jp0762525

(23) Zhang, G.; Mann, D.; Zhang, L.; Javey, A.; Li, Y.; Yenilmez, E.; Wang, Q.; McVittie, J. P.; Nishi, Y.; Gibbons, J.; et al. Proc. Natl. Acad. Sci. U. S. A. 2005, 102, 16141. doi: 10.1073/pnas.0507064102

(24) Zhang, G.; Qi, P.; Wang, X.; Lu, Y.; Mann, D.; Li, X.; Dai, H. J. Am. Chem. Soc. 2006, 128, 6026. doi: 10.1021/ja061324b

(25) Hardeman, D.; Esconjauregui, S.; Cartwright, R.; Bhardwaj, S.; D'Arsié, L.; Oakes, D.; Clark, J.; Cepek, C.; Ducati, C.; Robertson, J. J. Appl. Phys. 2015, 117, 044308. doi: 10.1063/1.4906846

(26) Chen, Y.; Wang, B.; Li, L.-J.; Yang, Y.; Ciuparu, D.; Lim, S.; Haller, G. L.; Pfefferle, L. D. Carbon 2007, 45, 2217. doi: 10.1016/j.carbon.2007.06.022

(27) Araujo, P. T.; Maciel, I. O.; Pesce, P. B. C.; Pimenta, M. A.; Doorn, S. K.; Qian, H.; Hartschuh, A.; Steiner, M.; Grigorian, L.; Hata, K.; et al. Phys. Rev. B 2008, 77, 241403. doi: 10.1103/PhysRevB.77.241403

(28) Zhang, D.; Yang, J.; Li, Y. Small 2013, 9, 1284. doi: $10.1002 / \mathrm{smll} .201202986$

(29) Ding, L.; Zhou, W.; Chu, H.; Jin, Z.; Zhang, Y.; Li, Y. Chem. Mater. 2006, 18, 4109. doi: $10.1021 / \mathrm{cm} 061122 \mathrm{e}$ 